\title{
The design of the slow-moving system of the new cave building based on the concept of "slow city"
}

\author{
Guorong Wang ${ }^{1, \mathrm{a}}$, Ting zhang ${ }^{1, \mathrm{~b}}$, Rong Shi ${ }^{* 1, \mathrm{c}}$, Bogang Li ${ }^{1, \mathrm{~d}}$ \\ ${ }^{1}$ Lanzhou University of Technology School of design art Lanzhou, China
}

\begin{abstract}
This paper fully discusses the design method of the slow-moving system of new cave buildings based on the concept of "slow city" by various modes of passage and road design, slow-moving system plant design, landscape node design, ecological facilities and so on. Cave architecture is an important part of native architecture, mainly distributed in china's loess plateau area, which is an ancient form of living. With the development of the times, the new cave building is still full of vitality. Based on the concept of "slow city", the design method of construction creates a slow-moving system into its landscape construction. The design follows the principles of regionality, ecology, humanization and emotion.
\end{abstract}

\section{Cave group landscape base on the concept of "Slow city"}

Cave dwelling is an architectural form that has been brilliant in the history of Chinese architecture, and it is the embodiment of the construction wisdom of the Chinese people. The mellow and unsophisticated shape of cave dwelling, with the ingenious use of hills, slopes and mountains, is an ideal architectural type that integrates with the environment and nature to maintain the balance between ecology and life (Figure 1). With the rapid development, this type of architecture is gradually fading out of people's sight. Nowadays, many scholars and experts are committed to restoring, innovating and developing this charming art that should exist on the land of China. It will upgrade its architecture and landscape comprehensively, develop new business forms, and create a green development combining tourism, health care and experience.

The ecological, cultural, green, healthy and sustainable environmental design of the new-type cave group conforms to the development mode of slow city concept. The term "slow city" originated from Italy's slow food culture in the 1990s, followed the slow city movement [1], advocating a slower pace of life and integration into the local culture, return to nature, as this concept is recognized. The concept of slow life in Slow city coincides with the current pace of rural life that Chinese people yearn for. As a basic component of Slow city, "slow moving" system is the link between rural life and natural forests.

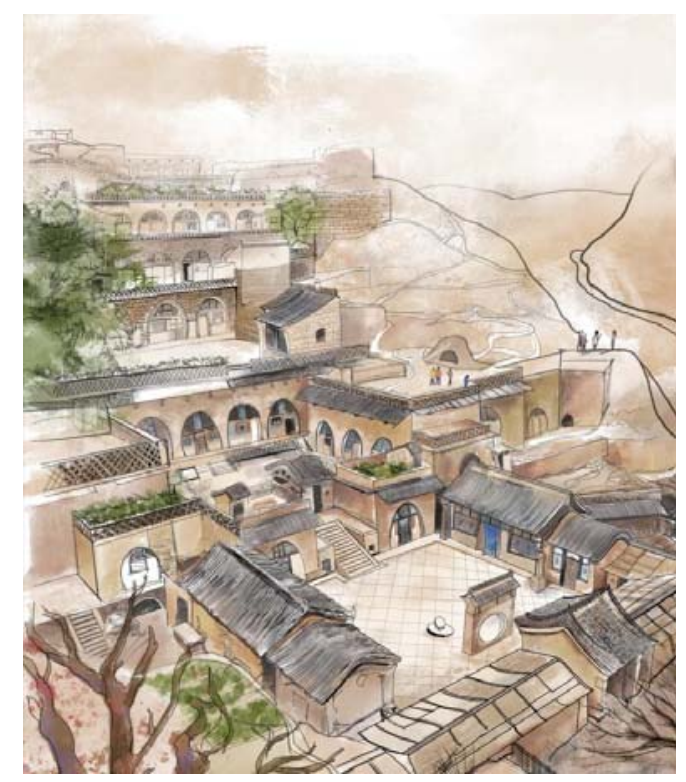

Figure 1. Traditional cave

\section{The modern slow-moving system based on four principles}

Slow-paced is a direct way for people to contact with the environment, which should be fully considered in landscape planning [2]. There are many experiences about the research and construction of greenways and slow lanes at home and abroad, but few truly play their role, especially in northwest China. Most of them still believe that bicycle lanes and relatively wide sidewalks should be added to the slow lane system, which seems to return to the concept of the bicycle era. Slow-moving system is not only the construction of "capillary type" slow-moving

\footnotetext{
ae-mail address: wang.kf@126.com

be-mail address: 854872108@qq.com

c* Corresponding author: 25204889@qq.com

de-mail address: 915449392@qq.com
} 
traffic network, convenient for walking and cycling, but also contains a series of landscape elements such as plant greening and landscape facilities, so as to achieve a healthy, dynamic and characteristic humanized lifestyle.

Healthy life, healthy industry and healthy environment constitute the core of a slow life. The cave dwelling group on the Loess Plateau in northwest China shows the local regional characteristics, in which people integrate into nature and conform to the local architectural form and way of life. Therefore, the design of the new type of slowmoving cave group system should follow the principles of regionalism, ecology, humanization and emotion.

\subsection{The design of the new-type cave group slow- moving system follows the principle of regionalism}

Regionalism is the concentrated reflection of local spirit and local feelings. The region where the loess Plateau cave is located is the geographical background of the formation of culture, as well as the concrete manifestation of the psychological structure formed by people's living style, behavior habit, value concept and communication mode in a specific region for a long time, which is the historical and cultural characteristics of this region. This context should be inherited in the design of the cave group landscape slow motion system.

All regions of cave group are connected by roads, which are designed by local residents for the purpose of a convenient life, and are the result of coordinated development between people and region. In the road design of slow-moving system, residents' understanding of the place should be respected, instead of being separated from the connection between local residents and special geographical environment. The road spontaneously created by people should be retained and properly optimized.

The design of the new cave group slow-moving system should protect the historical sites and preserve the remaining cave buildings. And then, the design should start from the two directions of the renovation and restoration of the original cave buildings and the modernization of the cave buildings, retaining the quaint architectural forms at the same time, with modern architecture means to reinforce the building foundation, solve architectural space ventilation and lighting conditions.

Native tree species have strict regional and unique community structure, complex ecosystem and natural landscape characteristics, and are the component unit of regional vegetation [3]. The protection and utilization of excellent native tree resources is of great significance to the characteristics of regional development and the sustainable development of road landscape [2].

From various elements and various forms of historical and cultural elements, regional sculptures, guideboards, art installations, etc., regional cultural elements are integrated to fully display regional characteristics. The art installation with regional cultural elements in the landscape can attract people's interest during the tour, interpret regional cultural characteristics, and explore the historical context in depth, so as to fully express the functional element of "slow walking".

\subsection{The design of the new slow-moving system for cave groups follows the ecological principle}

The Loess Plateau is one of the regions with the most serious soil erosion and the most fragile ecological environment in the world. The loess plateau has weak erosion resistance and is prone to gravity erosion under the effect of groundwater immersion and lubrication, forming a special topography with ravines and ravines. The ecology reveals the value of setting up the slow-moving system and the cave group. Modern regional construction planning focuses on the maximization of ecological benefits to achieve good environmental effects and create a good ecological system. The design of a new type of slow-moving system for cave groups should have a good ecological environment or function of maintaining and restoring the ecology.

The cave landscape is attached to the crisscrossing ravine of the loess plateau. The cave building group and the body background coordinate to form a unique skyline contours, and a slow-moving system connects the building groups with the landscape, forming a unique landscape system based on the topography and characteristics. At the same time, differentiation is emphasized in its construction, and flexible intervention of different landforms and landforms is adopted to avoid ecological damage [4].

The slope is a more prominent contradiction between people and the environment. The slope of slow-moving roads, plants, facilities and structures are different from the simple ornamental significance. To meet the multikinetic energy conditions such as traffic safety and ecological protection, pollution-resistant and noiseresistant tree species should be planted on the slope on both sides of the road.

Soil erosion and landslides are easily caused by slopes in large slope area. The stability of slopes should be considered in the design of road, solid slope and retaining wall. The thickness of soil layer, slope and slope origin are the basis for selecting specific methods.

\subsection{The design of the new slow-moving cave system follows the principle of humanization}

The service object of road traffic is human, and it should follow the people-oriented concept, fully considering people's physiological and psychological needs, and creating a safe, comfortable, healthy and convenient environment [5].

Slow traffic is the most concentrated embodiment of the road traffic system construction of the human requirements. In the landscape of cave dwellings in the Loess Plateau, various kinds of roadways converge into a road system, which bears all activities and traffic and has the closest relationship with people. When designing slow-moving transportation systems, the needs of residents must be understood first. That is to meet the basic traffic needs and ensure the physical and 
psychological safety of pedestrians. Pedestrians can communicate with each other in slow traffic and they can be physically and mentally happy in slow traffic [6].

In order to design a humanized slow-moving system, it is necessary to focus on analyzing the characteristics of activity of users and make specific analysis and differential measurement of the actual needs of different participants according to the activity characteristics. Due to the cave landscape and road space, the tour way can be divided into: walking activities and bicycle activities. The characteristics of activity of different age groups are not uniform and conclusive. For example, the vulnerable groups should take special treatment in their travel and cannot be isolated in road planning, thus causing psychological burden to this group (Figure 2).

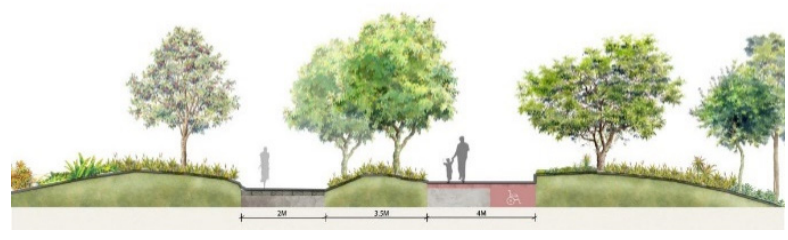

Figure 2. Walk to combine with bike paths

\subsection{The design of the new slow-moving cave system follows the emotion-oriented principle}

The most important purpose of cave landscape is to arouse viewers' emotional resonance and release their emotions through image expression. The road landscape must be combined with regional culture and folk custom characteristics, and the regional cultural characteristics should be integrated into the road landscape design.

Small scenery of the return to the nature of the wild interest, the beauty of the forest natural scenery step by step, wich makes full use of the original natural landforms suitable for mountains and rivers, the abundant features of green plants, and the characteristic traditional culture of loess Plateau mountains, so as to make the landscape move with each step [4].

In the road landscape design of slow-moving system, attention is fully paid to the creation of small scenery, which is an indispensable carrier in the road landscape. The shaping of small scenery has such strong regional characteristics, artistic appeal and strong affinity, reflecting the humanistic feelings, that people unwittingly slow down.

\section{Design and research of new type cave landscape slow motion system}

The Loess Plateau is a unique type of geomorphic landscape, which includes plateau, mound, gully and other geomorphic forms. Cave groups take the form of stepped yards down from the mountain. In the slow-moving system, the roads connect the caves to form small community relationships, which are attached to the trend of the mountain.

\subsection{Separation of lanes}

The travel modes for local residents and tourists can be divided into three forms: motor vehicles (private passenger cars, agricultural vehicles), non-motor vehicles (bicycles, handcarts, moped) and walking. Enter the entrance area of cave group and set up a motor vehicle parking lot in a relatively flat area. Each mountain forms a smaller group, and each group sets up a separate parking lot to facilitate the travel of local residents, and separate motor vehicles from non-motor vehicles with relatively slow driving speed and walking.

\subsection{Various traffic modes and road design}

(1) According to the particularity of mountainous topography on the Loess Plateau, caves are located among the mountains. Due to large slope, single vegetation coverage, and increased surface runoff caused by elevation difference, these areas are subject to soil and water loss and landslide. Traditional slope vegetation increases quickly in storm runoff, the effect of water seepage, water storage capacity, thus to cascade design of slope, slope by enhancing administrative levels, to slow down the rain flow velocity (figure 3 ), into every level of storm sewer corridor, plant furrow are installed near the road, as a last line of defense, the flow path of rainwater storage, and abundant slow landscape environment of the system. To avoid the discomfort to the road, the walkway should have enough lateral slope to realize the drainage function of the road, and the maximum lateral slope should not exceed 2\% (Fig.4).

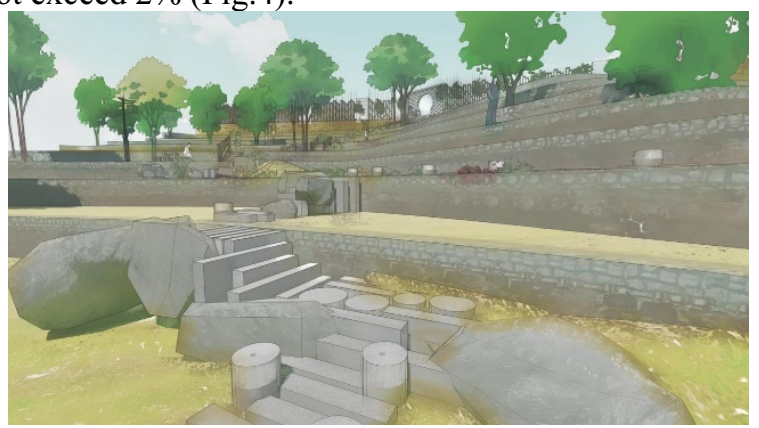

Figure 3. Steps and trails enhance the terrain experience

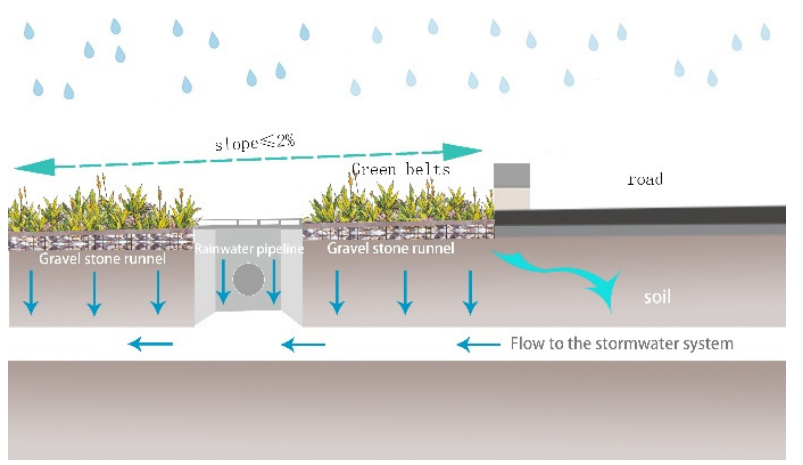

Figure 4. Drainage and storage of green areas beside roads

(2) Zigzag slope is the main road connecting the whole new cave-building complex. The road winding upwards reduces the road slope by less than $7 \%$ due to the height 
difference, which leads to the longer distance. Steps and Bridges should be set in the area with a large height difference to enhance the experience of the terrain and various convenient ways of passage (FIG. 3). A retaining wall is set at the steep slope (slope (non-vertical section) greater than 1:3) to enclose the planting space in layers. Where the slope is gentle (slope less than 1:3), stones are placed locally to solidify the soil, or planting with enclosed soil replacement under the forest. At the mountain vertical section, according to the mountain situation, retaining walls are built, beautifying the mountain section and ensuring safety at the same time.

(3) A horizontal pedestrian stop area is provided every 100 meters on the long slope of the walkway. Where steep slopes are unavoidable, other passages should also be provided [7]. The steps and trestle bridge can shorten the passage distance appropriately, can be used as a place for a short rest and stay to relieve the anxious psychology of being eager to get to the destination appropriately, and become the landscape node for people to communicate.

(4) Walkways barrier-free width should be not less than $1.5 \mathrm{~m}$. If the width of the foot path is not enough, the irregular giveway space should be built every 3 meter along the walking way, with the surrounding landscape to form unique ornamental and rest space. The surface of the walkway should be selected for greater friction. Because the surface is rougher close to the natural properties of the material, it will not be stranded by rain and become slippery, resulting in safety hazards.

(5) Different pavement materials and colors can clarify the division of road right, rather than directly dividing the road into two roads used by healthy people and disabled people respectively. Such a design can better reflect the humanistic care for slow travelers and improve safety and psychological comfort.

\subsection{Plant design of slow-moving system}

Road plant landscape effect should not be exaggerated and eye-catching in the location, shape, plant collocation and color design; instead, it should be appropriate to the main scene. Linear road, the line of sight is more open, with the landscape in the middle and far. When vehicles enter the area of cave group, open design is carried out to widen the visual field and introduce distant landscape and geomorphology into the line of sight, showing the overall features and scenery of this area. The plants in the close shot area are diversified, and the colors can be more gorgeous, which enhances the staggered occlusion relationship between the plant groups, guides the line of sight and reduces the driving speed of vehicles. The rest facilities and observation platforms are arranged in the mountainous areas. Barren and resistant plants are selected to enrich the vegetation types. Natural and mixed planting methods are adopted to enhance the sense of rhythm and form the landscape sequence [8].

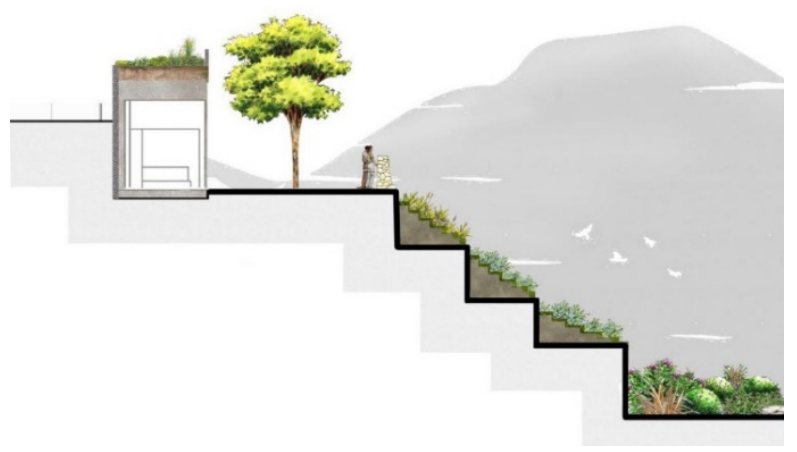

Figure 5. The anchoring effect of stepped slopes and plant

Plants not only play an ornamental role in the new-type cave group landscape in loess Plateau, but also play a special ecological system. It can better solve the erosion of loess soil caused by excessive surface runoff, internal collapse, effective drainage facilities and anchorage effect of plants, which can prevent the soil slope from sliding sideways due to insufficient bearing capacity, causing damage to the cave group buildings and facilities.

The selection of plants is the key factor for the success of soil-fixing slope protection to achieve the desired effect. For plants with developed roots and fast growth, root splitting will gradually destroy the rocks and soil mass of the slope and increase the cracks. When the slope angle is large, plants suitable for planting in this region with slow root growth and strong climate adaptability should be selected. Plants with underdeveloped roots will not have an impact on the soil weight. Plants with thick roots will increase the soil weight after the roots absorb water, and large slope areas are prone to collapse and landslide.

\subsection{Landscape node design}

The slow-moving system of road landscape nodes can create a rich stay space, promote random communication, and enhance the taste of sightseeing. The setting of landscape nodes should consider two elements: environmental elements and behavioral elements which influence each other and cannot be separated.

Environmental elements first consider the terrain and landform of the node area. In the mountainous terrain of the Loess Plateau, the landscape value of nodes and markers is not only reflected in the site space and the form of structures, but also the background of the mountain environment [9], for different terrain to create a unique landscape for this region.

The lines of the ridge act as linear signposts, and the twists and turns of the road setting do not cause visitors to lose their way and they try to find a way to travel. Moreover, the ridge, with an outward looking view, is the best terrain for pavilion gallery and observation platform construction, and serves as the observation node of the overall features of the cave group, so that the internal and external landscape of the cave building can echo each other and form a three-dimensional landscape network [10]. This place is suitable for sight-seeing, commemorative landmark or sculpture.

A closed concave terrain and inverted is the ideal natural stage, sun slope, topography of temperature rise, 
less sandy and warm compared with other terrain. Also, it is a pleasant climate area, setting up road connection point. Slow system on the design of the shop is to cancel the directional and instructive. Pedestrians spontaneous stay, feel the time increase, with large cave construction as the main body, the configuration of humanistic landscape, as the rest.

The line of sight of the slope is open on one side, the cave buildings are stacked and extended according to the mountain, and the landscape effect is strewn at random. It is a landmark building of group type and can be used as the most representative landscape node.

\subsection{Ecological design}

(1) Solar landscape lighting, solar street lamps, solar lawn lights and solar street signs are used in this design.

(2) On both sides of the road, small constructed wetlands are used in combination with landscape water for storage, purification and sewage treatment. Through ecological purification treatment of domestic sewage in the area, natural drainage is used to obtain intermediate water, which is reused to realize intensive utilization of water resources [11]

(3) The use of environmental protection materials can be broken and decomposed for secondary use after reaching the service life, without generating construction waste or harmful substances.

\section{CONCLUSION}

The design of a new type of cave landscape slow-moving system based on the concept of "slow city" is a natural link between people, natural landscape environment on the Loess Plateau and cave architecture. Therefore, based on the design principles of regionalism, ecology, humanization and emotion, the lane is stripped from the slow-moving area to enrich the slow-moving traffic structure of the loess Plateau mountainous terrain. The combination of slope step-type reconstruction and walking path solves the ecological problem and enhances the landscape gradation of the new-type cave group slowmoving system. Plants in slow-moving system guide slow-moving behavior through morphological design and reasonable distribution, solidify sloping land and maintain ecology. The landscape nodes of the slow-moving system cater to the terrain to create the unique landscape characteristics of the region, and the ecological design improves the construction height of the slow-moving system of cave groups. Finally, the slow-motion system is reasonably integrated into the landscape construction of the new cave group.

\section{Acknowledgment}

The work was financial supported by the research of A Holistic Study of the historical town form in Gansu Section of the Silk Road (51668039),2017.01-2019.12.

\section{References}

1. Wu Tong. Research on the Construction of "International Slow city" in Wufeng County [D]. Central South University for Nationalities,2017.

2. Gu Xiaoxu. Research on key Technical Indexes of modern Rural urban Road Landscape [D]. Chang 'an University,2012.

3. Zhang Yamei. Applied Research on native tree species in urban landscaping $[\mathrm{J}]$. Private Science and Technology,2013(12):213.

4. Zhao Ruifeng. Application exploration of Rural Landscape Design in Chongqing and West under the Concept of Slow Life [D]. Sichuan Academy of Fine Arts, 2018.

5. Sun Hongtao. Research on slow Traffic Organization in Xi 'an Under the low-carbon Concept [D]. Xi 'an University of Architecture and Technology,2011.

6. Wang Jing. Research on Greenway Slow Traffic System Planning and Design under human Dimension [D]. Anhui Agricultural University,2014.

7. Shi Chengyong. Toward rural Cities: A Study on the Landscape Ecological Model and Planning Strategy of Yangling Demonstration Area [D]. Xi 'an University of Architecture and Technology,2017.

8. Jiang Yujie. Study on road Landscape Design guided by typical geomorphic features in Altay, Xinjiang [D]. Chinese Academy of Forestry sciences, 2018.

9. Cao Ke, LI Heping, XIAO Jing, Chen Liang.Study on the Method of Mountain Urban Landscape Design based on the Mutual Contract between man and nature -- A Case study of urban design in Beibei New District, Yunyang County, Chongqing $[\mathrm{J}]$. Urban Planning, 2008,42(07):52-60.

10. Cao Ke. Theory and Method of Regional Adaptability of mountain Urban Design [D]. Chongqing University,2016.

11. Liu Song. Research on content System of Urban Landscape Water System Planning [A]. Chinese Society of Landscape Architecture. Chinese Society of Landscape Architecture: Chinese Society of Landscape Architecture,2011:4. 